\title{
Fluctuations in phenotypes and genotypes within populations of Pseudomonas aeruginosa in the cystic fibrosis lung during pulmonary exacerbations
}

\begin{abstract}
Correspondence
Craig Winstanley

c.winstanley@liv.ac.uk
\end{abstract}

Received 8 September 2009

Accepted 12 December 2009

\author{
Joanne L. Fothergill,, ${ }^{1,2}$ Eilidh Mowat, ${ }^{2}$ Martin J. Ledson, ${ }^{3}$ \\ Martin J. Walshaw ${ }^{3}$ and Craig Winstanley ${ }^{1,2}$ \\ ${ }^{1}$ Biomedical Research Centre, Royal Liverpool University Hospital, Liverpool L69 3GA, UK \\ ${ }^{2}$ Division of Medical Microbiology, School of Infection and Host Defence, University of Liverpool, \\ Liverpool L69 3GA, UK \\ ${ }^{3}$ Regional Adult Cystic Fibrosis Unit, Liverpool Heart and Chest Hospital, Liverpool L14 3PE, UK
}

\begin{abstract}
Chronic respiratory infection by Pseudomonas aeruginosa contributes significantly to the morbidity and mortality associated with cystic fibrosis (CF). Using a series of phenotypic and genotypic tests on collections of 40 isolates per sputum sample, we analysed fluctuations within sputum populations of the $P$. aeruginosa Liverpool epidemic strain (LES) during pulmonary exacerbations. For each of three patients, three sequential sputum samples were analysed: (1) on presentation with exacerbation at the Regional Adult Cystic Fibrosis Unit, Liverpool; (2) a few days into intravenous antibiotic treatment; (3) when the patient had recovered. Fluctuations were observed in morphotype distribution, the production of virulence-associated quorum-sensingdependent exoproducts (the phenazine compound pyocyanin and the elastase LasA), antibiotic susceptibility profiles and levels of auxotrophy. PCR assays were used to screen isolates for the presence of novel regions of the LES genome (islands and prophages) and to detect free phages. In one patient there was an increase in the prevalence of the LESGI- 5 genomic island during the sampling period from 10 to $97.5 \%$ carriage. LES phages 2-4 were detected in either the majority or all sputum samples tested, indicating widespread phage activity during the sampling period. The results of this study are indicative that significant fluctuations occur within $P$. aeruginosa populations during short periods of pulmonary exacerbation and intravenous antibiotic therapy.
\end{abstract}

\section{INTRODUCTION}

Pseudomonas aeruginosa is the most important pathogen infecting the lungs of cystic fibrosis (CF) patients and, once established, infections with $P$. aeruginosa are impossible to eradicate (Hart \& Winstanley, 2002). The Liverpool epidemic strain (LES) of $P$. aeruginosa is a particularly successful CF transmissible strain and is widespread in the UK (Scott \& Pitt, 2004). First identified in 1996 (Cheng et al., 1996), the LES has been associated with increased morbidity (Al-Aloul et al., 2004), transmission to both the non-CF parents of a CF patient (McCallum et al., 2002) and infection of a pet cat (Mohan et al., 2008). In addition, we have reported previously that some LES isolates exhibit an unusual phenotype characterized by enhanced antimicrobial resistance (Salunkhe et al., 2005) and overproduction of quorum-sensing-regulated products (Fothergill et al., 2007) with potential roles in pathogenicity in CF (Winstanley \& Fothergill, 2009).

Abbreviations: BSAC, British Society for Antimicrobial Chemotherapy; CF, cystic fibrosis; LES, Liverpool epidemic strain.
The genome sequence of an isolate of the LES, LESB58, indicated the presence in its accessory genome of five intact prophages (LES prophages 2-6) and five genomic islands (LESGIs1-5). A rat model of chronic lung infection was used to demonstrate the involvement of a novel genomic island (LESGI-5) and novel prophages (LES prophages 2, 3 and 5) in the in vivo competitiveness of the strain (Winstanley et al., 2009), implicating regions of the accessory genome in its success as a pathogen.

Exacerbations, described as recurrent episodes of increased pulmonary symptoms, occur periodically in CF (Goss \& Burns, 2007). Hospitalization during these periods is common and intravenous antibiotics are generally used, with the aim of reducing symptoms and increasing lung function (Regelmann et al., 1990). It is known that the characteristics of $P$. aeruginosa isolates isolated from $\mathrm{CF}$ patients change over long periods of time; mutations leading to mucoid conversion, auxotrophy, loss of motility and loss of virulence are common amongst CF isolates (Smith et al., 2006). However, little is known about the fluctuations within $P$. aeruginosa populations in the $\mathrm{CF}$ 
lung during very short periods of intense selective pressure, such as when intravenous antibiotic therapy is administered during exacerbations.

\section{METHODS}

Sputum samples. Sputum samples were collected from adult CF patients at the Regional Adult Cystic Fibrosis Unit in Liverpool. These represented routine samples taken for diagnostic purposes, and the antimicrobial therapy was determined by clinicians. For each patient, three samples were taken: the first at the beginning of the exacerbation, before intravenous antibiotic treatment had commenced (sample A); the second during the exacerbation, whilst antibiotic therapy was being carried out (sample B); the third at the end of the exacerbation when symptoms had resolved (sample C) (Table 1). Exacerbations were defined by a clear set of criteria, including a drop in $\mathrm{FEV}_{1}$ (forced expiratory volume in $1 \mathrm{~s}$ ), increased sputum or discoloration, temperature and increased cough (Goss \& Burns, 2007). All of the patients studied were chronically infected with the LES. Sputum was treated with an equal volume of Sputasol (Oxoid), incubated at room temperature with shaking at 200 r.p.m. for $15 \mathrm{~min}$, and then cultured on Pseudomonas selective agar with $\mathrm{CN}$ supplement (Oxoid). Forty single colonies were isolated per sputum sample, ensuring that each different colony morphology type was proportionately represented. Each of these 40 isolates was confirmed as $P$. aeruginosa LES using PCR tests for the amplification of the P. aeruginosa oprL gene (De Vos et al., 1997) and the two LES markers, PS21 (Parsons et al., 2002) and LESF9 (Smart et al., 2006), before being subjected to a range of phenotypic and genotypic tests (Fig. 1).

Simple phenotypic tests. Auxotrophy was determined by testing the ability of isolates to grow on glucose M9 media. Hypermutability was assessed by determining spontaneous mutation rates on Luria agar containing rifampicin (Sigma) $\left(300 \mu \mathrm{g} \mathrm{ml} \mathrm{ml}^{-1}\right)$ following overnight growth in Luria broth as described by Oliver et al. (2000). Colony morphology was assessed on Columbia agar plates (Oxoid).

Exoproduct production assays. Pyocyanin production was determined and cut-off values for overproduction were used as described previously (Fothergill et al., 2007). Overproduction refers to culture supernatant with an absorbance at $695 \mathrm{~nm}$ of $>0.1$ after overnight

Table 1. Sputum samples used in this study

Sample A was taken at the beginning of the exacerbation when the CF patients were admitted to the Regional Adult Cystic Fibrosis Unit with exacerbation. Sample B was taken during intravenous antibiotic treatment. Sample $\mathrm{C}$ was taken at the end of the exacerbation.

\begin{tabular}{|lccc|}
\hline Patient & Sample & Date & Antibiotic treatment \\
\hline I & A & $12 / 01 / 2006$ & Meropenem/colistin \\
& B & $16 / 01 / 2006$ & \\
II & C & $20 / 01 / 2006$ & \\
& A & $03 / 02 / 2006$ & Meropenem/colistin \\
& B & $06 / 02 / 2006$ & \\
III & C & $17 / 02 / 2006$ & \\
& A & $11 / 04 / 2006$ & Ceftazidime/colistin \\
& B & $13 / 04 / 2006$ & \\
& C & $21 / 04 / 2006$ & \\
\hline
\end{tabular}

growth of a strain in $5 \mathrm{ml}$ Luria broth with shaking at 200 r.p.m. LasA activity was determined using a spectrophotometric assay measuring the lysis of a staphylococcal (Staphylococcus aureus NCTC 6571) suspension (Kessler et al., 2004) on a plate reader (Fluostar omega; BMG Labtech). LasA overproduction was defined as a value at the end of the assay of $<80 \%$ of the original absorbance at $600 \mathrm{~nm}$.

Determination of antibiotic susceptibility. Antibiotic susceptibilities were determined by disc diffusion tests for six antibiotics (ceftazidime, colistin, ciprofloxacin, meropenem, tazobactam/piperacillin and tobramycin) using current British Society for Antimicrobial Chemotherapy (BSAC) guidelines (Andrews, 2001). The following amounts were used: $85 \mu \mathrm{g}$ tazobactam/piperacillin, $10 \mu \mathrm{g}$ meropenem, $10 \mu \mathrm{g}$ tobramycin, $5 \mu \mathrm{g}$ ciprofloxacin, $30 \mu \mathrm{g}$ ceftazidime and $25 \mu \mathrm{g}$ colistin sulphate (all from Oxoid). The sizes of the zones of inhibition $(\mathrm{mm})$ were also recorded and statistical analysis was performed using SPSS data analysis software.

PCR amplification assays. PCR amplifications were carried out in a total volume of $25 \mu \mathrm{l}$. For each reaction, $1.25 \mathrm{U}$ GoTaq polymerase (Promega), $1 \times$ TaqMaster (Helena Biosciences), $300 \mathrm{nM}$ each oligonucleotide primer (Sigma-Genosys; Table 2), $1 \times$ Taq buffer, $2.5 \mathrm{mM} \mathrm{MgCl}_{2}$ and $100 \mu \mathrm{M}$ nucleotides (dATP, dCTP, dGTP, dTTP) was used along with $1 \mu \mathrm{l}$ DNA from boiled suspensions of colonies. PCR amplification was carried out for 30 cycles of $95{ }^{\circ} \mathrm{C}(1 \mathrm{~min})$, the chosen annealing temperature $(2 \mathrm{~min})$ and $72{ }^{\circ} \mathrm{C}(2 \mathrm{~min})$. Following this, a final extension step of $72{ }^{\circ} \mathrm{C}$ for 10 min was carried out. In the case of LES prophages 2, 3, 4 and LESGI-5, additional PCR assays were performed using primers designed for the flanking regions of the insertions, and therefore only if the insertion was not present would the expected band be observed. For PCR amplification to detect free phages directly from sputum samples, the sputum was first treated with Sputasol, diluted $(1: 10)$ with sterile distilled water, filtersterilized $(0.2 \mu \mathrm{m})$ and DNase I treated as follows: addition of $3.5 \mathrm{U}$ DNase I (Roche), $3.5 \mu \mathrm{l} 10 \times$ DNase I buffer and $3 \mu$ sterile distilled water, followed by incubation for $15 \mathrm{~min}$ at room temperature. To stop the reaction $3.5 \mu \mathrm{l} 25 \mathrm{mM}$ EDTA ( $\mathrm{pH} 8.0$ ) was added and the solution was heated to $65{ }^{\circ} \mathrm{C}$ for $10 \mathrm{~min}$. Following this, PCR amplification (Table 2) was carried out using $5 \mu \mathrm{l}$ of the phage preparation. Each PCR amplification reaction was replicated twice.

\section{RESULTS AND DISCUSSION}

We investigated the changes in the prevalences of various phenotypes and genotypes amongst $P$. aeruginosa LES populations during short periods of exacerbation and intravenous antibiotic therapy. The total number of $P$. aeruginosa recovered from the sputum samples ranged from $1 \times 10^{3}$ to $1 \times 10^{6}$ c.f.u. $\mathrm{ml}^{-1}$. However, there was no clear pattern in the change in the number of $P$. aeruginosa recovered from sputum following treatment with antibiotics. For patient $\mathrm{I}$, there was a marked reduction in $P$. aeruginosa between samples $\mathrm{A}$ and $\mathrm{C}\left(1 \times 10^{6}\right.$ to $3 \times 10^{4}$ c.f.u. $\left.\mathrm{ml}^{-1}\right)$; for patient III, the numbers remained relatively constant, at around $5 \times 10^{5}$ c.f.u. $\mathrm{ml}^{-1}$; for patient II the number of $P$. aeruginosa actually increased over the exacerbation period, from $1 \times 10^{4}$ c.f.u. $\mathrm{ml}^{-1}$ in sample A to $7 \times 10^{5}$ c.f.u. $\mathrm{ml}^{-1}$ in sample C. In order to facilitate multiple testing, we restricted our study to 40 isolates per sputum sample and to 3 CF patients. Whilst we accept that these numbers may not be sufficient to fully represent the variation present, our study was conducted in considerably more depth than would normally be used in 


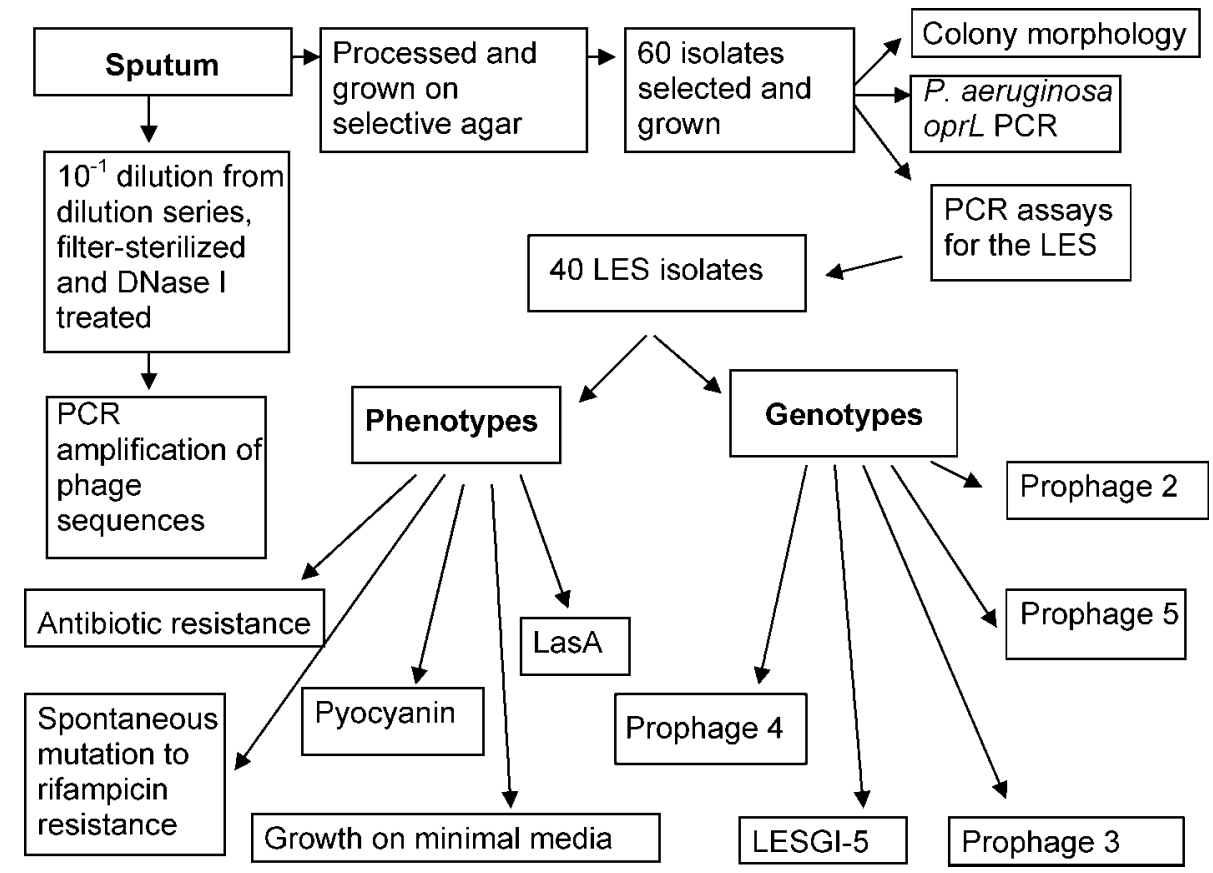

Fig. 1. Processing of the sputum samples and tests performed.

the analysis of sputum samples in diagnostic laboratories. All of the isolates tested were identified as the $P$. aeruginosa LES by virtue of PCR-positive assays for oprL and the two LES markers (PS21 and LESF9).

\section{Changes in the phenotype of $\boldsymbol{P}$. aeruginosa LES populations during the exacerbations}

For patients I and II, there was an increase in the prevalence of isolates exhibiting overproduction of pyocyanin and LasA during the exacerbation period (Table 3). Conversely, isolates from patient III showed a very different trend, beginning with a higher level of overproducers $(37.5 \%)$, which gradually decreased over the exacerbation period (to $15 \%$ ). In all samples, pyocyaninnegative isolates could also be identified, with the level in the population ranging from 20 to $67.5 \%$. There was strong concordance between levels of LasA activity and pyocyanin production (Table 3 ).

There was a general trend in the changes in colony morphologies. At the beginning of the exacerbation, in all three patients, the majority of isolates were green, smooth and non-mucoid (Fig. 2). However, the prevalence of this morphotype decreased over the exacerbation period and the occurrence of both white, mucoid isolates and smooth, non-mucoid, white/straw-coloured isolates increased. A very low level of mucoid translucent isolates was also detected in samples IB and IIB. In all samples, the majority of isolates were non-mucoid rather than exhibiting the mucoid phenotype commonly associated with chronic infection (Fig. 2). All of the pyocyanin overproducers had green pigmented colony morphology, suggesting that the colour observed was at least in part due to the high levels of pyocyanin produced. However, many of the other isolates were also pigmented and it was unclear whether this was due to the production of pyocyanin or other pigmented products such as pyoverdine.

Changes in the antibiotic susceptibility profiles were detected in all of the patients during the exacerbation period (Table 3). The overall prevalence of resistance to tobramycin was very different between patients I and II, but varied little between the three samples taken for each patient. In contrast, there was a decrease in the prevalence of isolates from patient III with resistance to tobramycin over the sampling period. The LES populations in all three patients showed some decrease in the prevalence of resistance to ceftazidime during the exacerbation period, with the greatest decrease observed in patient III (from $97.5 \%$ resistance to $27.5 \%$ resistance). It is interesting to note that, during this period, patient III was treated with ceftazidime. In contrast, the two patients treated with meropenem (patient I and II) showed a greater prevalence of resistance to meropenem at the end of the treatment compared to the beginning (Table 3 ).

The change in prevalence of resistance to tazobactam/ piperacillin was patient specific and no overall trend could be observed. This varied from very little change for patients I and III, to a marked decrease in the prevalence of resistance for patient II. In all three patients, the prevalence of isolates with resistance to ciprofloxacin increased during the sampling period (Table 3 ). 
Table 2. Oligonucleotide primers used in this study

'Circular genome' indicates that these PCR assays target the phage in its circularized genomic form only and will not yield amplicons with linear prophage DNA inserted into the bacterial genome.

\begin{tabular}{|c|c|c|c|c|}
\hline Name & Sequence $5^{\prime} \rightarrow 3^{\prime}$ & Size $(b p)$ & Target gene & Reference \\
\hline PAL1 & ATGGAAATGCTGAAATTCGGC & \multirow[t]{2}{*}{504} & \multirow[t]{2}{*}{ oprL } & \multirow[t]{2}{*}{ De Vos et al. (1997) } \\
\hline PAL2 & CTTCTTCAGCTCGACGCGACG & & & \\
\hline PS21R & AAAACGTAGCAAGCAGTG & 364 & PS21 (LES marker) & Parsons et al. (2002) \\
\hline LESF9F & AACACTTGCTCCATCTGC & \multirow[t]{2}{*}{431} & \multirow[t]{2}{*}{ LESF9 (LES marker) } & \multirow[t]{2}{*}{ Smart et al. (2006) } \\
\hline LESF9R & CACGATATCCAGCAAGAC & & & \\
\hline LES1R & TGAAGCCGACGATGGAAG & 392 & LES prophage 2 & Smart et al. (2006) \\
\hline 4138up & TAGCCAGCGCATCAGCAT & \multirow[t]{2}{*}{998} & \multirow{2}{*}{$\begin{array}{l}\text { tyrS and PALES_07881 (PA4139) flanking } \\
\text { regions of LES prophage } 2\end{array}$} & \multirow[t]{2}{*}{ This study } \\
\hline 4139up & TTCATCGAAGCTCAGTTC & & & \\
\hline LES1out & GACTCAGGAGATCACGAA & \multirow[t]{2}{*}{425} & \multirow[t]{2}{*}{ LES prophage 2 (circular genome) } & \multirow[t]{2}{*}{ This study } \\
\hline LES1down & CAAGGGTGCGAGAGTATC & & & \\
\hline LES1nestF & TTTGGTGATGATCGGCTTAGC & 289 & $\begin{array}{l}\text { LES prophage } 2 \text { (circular genome); using } \\
\text { nested primers } \\
\text { LES prophage } 3\end{array}$ & This study \\
\hline 3664 & TTTGATGCCGTAGAGAAC & 363 & $\begin{array}{l}\text { PALES_13721 (PA3663) and PALES_13201 } \\
\text { (PA3664) flanking regions of LES } \\
\text { prophage } 3\end{array}$ & This study \\
\hline Cluster6up & TTCGGCATCTGGTCGTTC & \multirow[t]{2}{*}{532} & \multirow[t]{2}{*}{ LES prophage 3 (circular genome) } & This study \\
\hline Cluster6dn & GCTGCCAGGCTTCATGAA & & & \\
\hline Clus6nestF & GGATCGACGTGGCATAATCTG & 410 & LES prophage 3 (circular genome); using & This study \\
\hline Clus6nestR & ACGATTCTCCGGCATGCAGCG & & nested primers & \\
\hline D3112cIF & GACAGTTACGCCTATATC & 383 & LES prophage $4 \mathrm{cI}$ repressor gene & This study \\
\hline D3112cIR & ATGATGTCAACTGCTTCC & & & \\
\hline $3464 d n$ & ACTTCAGCGAGAGCAACCA & 722 & LES prophage $4 \mathrm{cI}$ repressor and flanking & This study \\
\hline D3112cIout & GGAAGCAGTTGACATCAT & & gene PALES_15481 (PA3464) & \\
\hline D3112nestF & TGTATGACGCTCGGGTGA & 339 & LES prophage $4 \mathrm{cI}$ repressor gene; using & This study \\
\hline $831 \mathrm{~F}$ & TGCAATACCTGACCACCA & 310 & oruR and PALES_44861 (PA832) flanking & This study \\
\hline $831 \mathrm{R}$ & CAATGATGGTGCAGTTGC & & regions of LES genomic island-5 & \\
\hline
\end{tabular}

To determine whether the changes in resistance observed using the accepted BSAC cut-off values were due to significant shifts in the resistance of the population or simply fluctuations of inhibition zone size around the breakpoint, the zone size produced by each isolate to the six antibiotics was measured and used to calculate a mean for each sputum sample. The change in the mean zone size was monitored across the three samples to determine whether the population, as a whole, was becoming more or less resistant to the antibiotics. There was very good agreement between the trends in the changes in resistance as determined by using breakpoints compared to the change in mean zone sizes. As the prevalence of resistance increased, the mean zone size decreased. Examples of significant shifts in the mean zone size were observed for each of the antibiotics; however, for ceftazidime, meropenem and tobramycin these changes were significant between the first and final sample in all three patients $(P<0.05)$. Measuring the zone of inhibition also gave an indication of more subtle differences in the resistance. For example, the LES populations in both patient I and patient II would be classed as ceftazidime resistant using the breakpoint cut-off value $(23 \mathrm{~mm})$. However, the population in patient II appeared to be much more resistant, with a mean zone size of only $5 \mathrm{~mm}$ by sample $\mathrm{C}$, whereas the LES population from patient I had a mean zone size of $20 \mathrm{~mm}$.

The prevalence of auxotrophic mutants amongst the LES populations from two patients (I and III) increased from 
Table 3. Phenotypic characteristics of the 40 isolates taken from each sputum sample

Values indicate the percentage of the 40 isolates with each characteristic. Sputum samples were taken: at the beginning of an exacerbation (sample A), during the exacerbation and antibiotic therapy (sample B) and at end of the exacerbation (sample C).

\begin{tabular}{|c|c|c|c|c|c|c|c|c|c|c|}
\hline \multirow[t]{2}{*}{ Sample } & \multicolumn{2}{|c|}{ Overproduction $(\%)^{*}$} & \multicolumn{6}{|c|}{ Antibiotic resistance $(\%) \dagger$} & \multirow[t]{2}{*}{ AUX (\%) } & \multirow[t]{2}{*}{ HYP (\%) } \\
\hline & Pyocyanin & LasA & ТОВ & CEF & MEM & TZP & $\mathrm{CIP} \ddagger$ & CT & & \\
\hline IA & 10 & 10 & 22.5 & 97.5 & 12.5 & 17.5 & $7.5(70)$ & 0 & 0 & 5 \\
\hline IB & 32.5 & 30 & 20 & 77.5 & 85 & 25 & $50(45)$ & 0 & 30 & 10 \\
\hline IC & 27.5 & 27.5 & 20 & 67.5 & 55 & 27.5 & $57.5(40)$ & 0 & 57.5 & 10 \\
\hline IIA & 5 & 7.5 & 100 & 100 & 37.5 & 90 & $57.5(42.5)$ & 0 & 7.5 & 7.5 \\
\hline IIB & 47.5 & 47.5 & 95 & 97.5 & 27.5 & 37.5 & $42.5(57.5)$ & 0 & 2.5 & 10 \\
\hline IIC & 12.5 & 12.5 & 97.5 & 92.5 & 77.5 & 45 & $92.5(7.5)$ & 0 & 2.5 & 5 \\
\hline IIIA & 37.5 & 37.5 & 95 & 97.5 & 97.5 & 5 & $2.5(80)$ & 0 & 0 & 5 \\
\hline IIIB & 22.5 & 22.5 & 2.5 & 37.5 & 40 & 12.5 & $52.5(42.5)$ & 0 & 32.5 & 15 \\
\hline IIIC & 15 & 15 & 40 & 27.5 & 27.5 & 0 & 17.5 & 0 & 42.5 & 5 \\
\hline
\end{tabular}

AUX, Auxotrophy; HYP, hypermutability (based on spontaneous resistance to rifampicin).

* Overproduction of pyocyanin refers to isolates giving an absorbance at $695 \mathrm{~nm}$ of $>0.1$; overproduction for LasA activity refers to a value at the end of the assay of $<80 \%$ of the original absorbance at $600 \mathrm{~nm}$ for $S$. aureus suspensions.

$\dagger$ The antibiotics tested were tobramycin (TOB), ceftazidime (CEF), meropenem (MEM), tazobactam/piperacillin (TZP), ciprofloxacin (CIP) and colistin (CT).

$\ddagger$ The percentage found to have intermediate resistance to ciprofloxacin is shown in parentheses.

undetectable to 57.5 and $42.5 \%$, respectively, during the sampling period (Table 3 ). In contrast the prevalence of auxotrophic mutants in samples from patient II remained low throughout. Isolates exhibiting mutation rates compatible with the hypermutable phenotype were identified in all of the samples, but prevalence levels varied little (5$15 \%$; Table 3).

In our study, all three patients were treated with dual intravenous antibiotic therapy of colistin and ceftazidime (patient III) or colistin and meropenem (patients I and II). Colistin has been shown to have an additive effect when used in conjunction with either ceftazidime or meropenem (Guzel \& Gerceker, 2008). However, serious questions have been raised generally about the levels of antibiotics reaching the lung during therapeutic intervention in $\mathrm{CF}$ (Moriarty et al., 2007). This could have consequences with respect to the development of resistance as well as the efficacy of therapy. In this study, changes were observed in the $P$. aeruginosa LES antibiotic susceptibility profiles during therapy. Although it is difficult to discuss general trends in a study of only three patients, whereas the two patients treated with meropenem showed an increase in the prevalence of isolates with resistance to meropenem, the patient treated with ceftazidime showed an overall decrease in the prevalence of isolates with resistance to ceftazidime. Hence, although one might expect an increase in the occurrence of isolates showing resistance to a therapeutic agent, this was not the case for all three patients in our study, indicating that levels of antibiotics reaching the target population may not be sufficient to select for a resistant subpopulation.
Interestingly, in all three patients, the proportion of isolates resistant to ciprofloxacin increased during therapy. Ciprofloxacin resistance occurs via two major resistance mechanisms: firstly, due to mutations in the DNA gyrase or topoisomerase genes; secondly, through activation of efflux pumps with ciprofloxacin being a substrate for the pumps MexAB-OprM, MexCD-OprJ, MexEF-OprN and MexXY-OprM. Both forms of resistance mechanisms can occur simultaneously during antibiotic treatment and this can result in the development of highly resistant isolates (Nakajima et al., 2002; Niga et al., 2005). Interestingly, it has been reported that treatment with drugs other than ciprofloxacin is a significant risk factor in the development of resistance to ciprofloxacin (Hyatt \& Schentag, 2000). However, we have no direct evidence that the increase in ciprofloxacin resistance is the result of increased mutations rather than selection for a subpopulation characterized by resistance to ciprofloxacin. It is interesting to note that no LES isolate was completely sensitive or completely resistant to all of the six antibiotics tested, and that the widespread use of colistin to treat LES-infected patients within our clinic appears justified by the lack of resistance observed amongst LES isolates in this study.

Diversity within each population with regards to a number of other phenotypic markers was also observed. It has been reported that during periods of exacerbation there is evidence of oxidative damage in the CF lung (McGrath et al., 1999). Endogenous oxidative stress is thought to generate diversity in $P$. aeruginosa populations by causing dsDNA breaks. The mutagenic repair of such breaks then leads to diversity (Boles \& Singh, 2008). The role of 

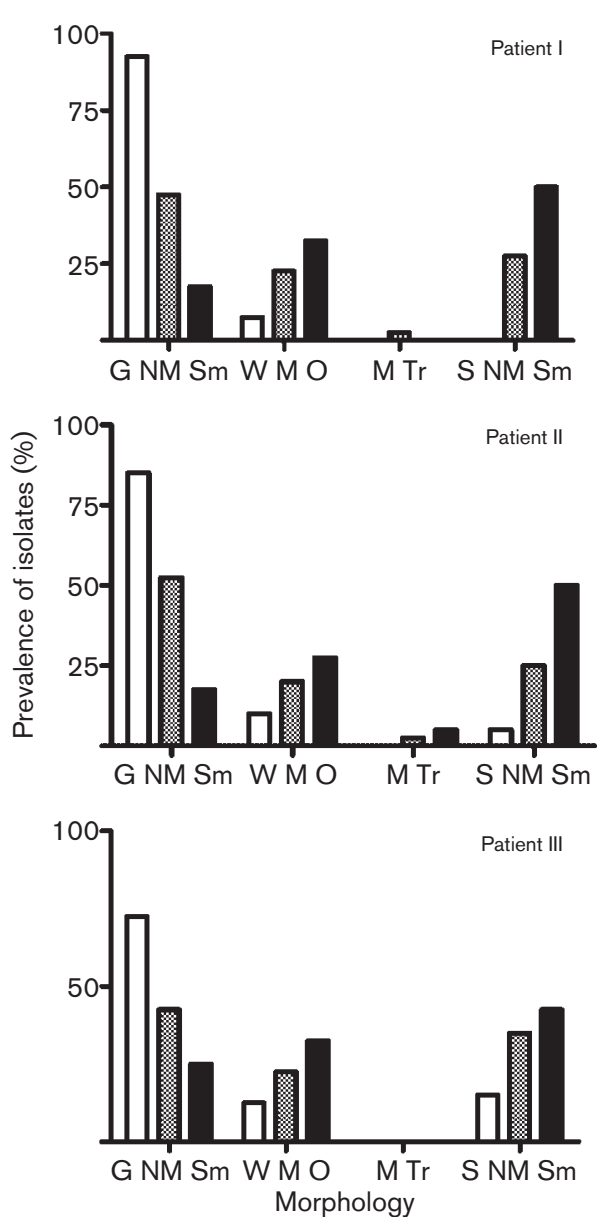

Fig. 2. Prevalence of colony morphotypes in the sputum samples. Percentage values are indicated for the following four colony morphology types: green, non-mucoid, smooth (G, NM, Sm); white, mucoid, opaque ( $\mathrm{W}, \mathrm{M}, \mathrm{O})$; translucent mucoid $(\mathrm{Tr}, \mathrm{M})$; straw-coloured, non-mucoid, smooth (S, NM, Sm). Results for three sputum samples per patient are shown, representing samples taken at the beginning of the exacerbation but before intravenous antimicrobial therapy (white bars) (sample A), samples taken during the exacerbation and antibiotic treatment (grey bars) (sample B), samples taken at end of exacerbation (black bars) (sample C).

oxidative stress was reinforced by experiments showing that the addition of antioxidants reduces DNA damage and bacterial diversity (Boles \& Singh, 2008). In this study, in all three patients, the colony morphology showed a consistent shift from a smooth, pigmented morphotype to an increase in white mucoid isolates and a general increase in the diversity of the bacteria with respect to morphology. Conversion into a mucoid phenotype during CF lung infection with $P$. aeruginosa has been widely reported. Mucoid isolates are thought to be more resistant to phagocytosis (Cabral et al., 1987) and antibiotics (Govan \& Fyfe, 1978). Furthermore, mucoid conversion and selection has also been associated with the presence of reactive oxygen species, which can be released by the host immune system (polymorphonuclear leukocytes) (Mathee et al., 1999).

It has also been suggested that the use of certain antibiotics can affect the quorum-sensing system (Skindersoe et al., 2008), potentially leading to increases in exoproducts, including pyocyanin, that may contribute to the presence of reactive oxygen species (Winstanley \& Fothergill, 2009). We detected LES isolates with the previously reported pyocyanin overproduction phenotype (Fothergill et al., 2007) in each of the sputum samples tested. The physiological relevance of this phenotype in the CF lung is unknown but it could potentially lead to increased oxidative effects in patients infected with the LES.

In previous studies, the level of hypermutability amongst CF isolates has ranged from 18 to $54.4 \%$ (Montanari et al., 2007; Ciofu et al., 2005). It has been shown that hypermutable isolates can be prevalent at high levels in chronic infections, such as the CF lung, compared to acute infections, where prevalence is less than $1 \%$ (Oliver et al., 2000). The presence of hypermutable isolates has been associated with accelerated resistance to antimicrobials, especially during exposure to antibiotics (Oliver, 2004; Plasencia et al., 2007). However, hypermutable isolates have been shown to have increased susceptibility to colistin (Macia et al., 2007), and therefore colistin could be an ideal choice in dual therapy to counteract any selection for resistance. In this study, the prevalence of hypermutable isolates remained constant throughout the sampling period (5-15\%), suggesting that any changes in antibiotic susceptibility or other phenotypes were not caused by the expansion of this subset of the LES population.

\section{Changes in the genotype of the $P$. aeruginosa LES populations during exacerbations}

PCR assays were performed using oligonucleotide primers for sequences specific to the four LES prophages that are not found in P. aeruginosa strain PAO1 (LES prophage 2, 3, 4 and 5) and the novel genomic island, LESGI-5 (Table 4). None of the LES isolates tested in this study contained LES prophage 5. However, all of the other LES prophage sequences assayed (from LES prophages 2-4) were detected in all of the LES isolates tested from patients I and II. Likewise, in patient III, LES prophage 2-4 sequences were detected in all isolates tested prior to therapeutic intervention (sample A). However, loss of LES prophages was detected in subsequent samples from patient III (Table 4). In sample C from patient III, LES prophages 2, 3 and 4 were all absent from the genomes of two isolates (Table 4). Prophage deletions were confirmed by PCR amplification using primers flanking the prophages in the genome of the LES.

In patient I, the prevalence of LESGI-5 amongst isolates increased from a level of 10 to $97.5 \%$ over the sampling period. However, in the other two patients the prevalence of LESGI-5 was high throughout (97.5-100\%). 
Table 4. Prevalence of LES prophages and LESGI-5 amongst LES isolates

The percentage of PCR isolates positive for the presence of LES prophages and LESGI-5 sequences amongst sets of 40 LES isolates are shown for sputum samples taken at the beginning of an exacerbation (sample A), during the exacerbation and antibiotic therapy (sample B) and at end of the exacerbation (sample C).

\begin{tabular}{|c|c|c|c|c|c|c|}
\hline Patient & Sample & Prophage $2(\%)$ & Prophage $3(\%)$ & Prophage $4(\%)$ & Prophage $5(\%)$ & LESGI-5 (\%) \\
\hline \multirow[t]{3}{*}{ I } & $\mathrm{A}$ & 100 & 100 & 100 & 0 & 10 \\
\hline & B & 100 & 100 & 100 & 0 & 65 \\
\hline & $\mathrm{C}$ & 100 & 100 & 100 & 0 & 97.5 \\
\hline \multirow[t]{3}{*}{ II } & A & 100 & 100 & 100 & 0 & 97.5 \\
\hline & B & 100 & 100 & 100 & 0 & 100 \\
\hline & $\mathrm{C}$ & 100 & 100 & 100 & 0 & 100 \\
\hline \multirow[t]{3}{*}{ III } & A & 100 & 100 & 100 & 0 & 100 \\
\hline & B & 100 & 97.5 & 100 & 0 & 100 \\
\hline & $\mathrm{C}$ & 92.5 & 95 & 95 & 0 & 100 \\
\hline
\end{tabular}

Overall, less variation was observed with respect to the genotypic markers targeted in this study compared to the phenotypic characteristics. LES prophages 2, 3 and 4 were identified in every sputum sample, but there was an example of loss of prophages from isolates within the population in one of the patients over the course of the study.

\section{Further screening of multiple sputum samples for prevalences of LESGI-5 and auxotrophy}

Following the results obtained from the screening of isolates from the three patients (I, II and III), we decided to carry out further screening to study changes in the prevalence of two markers for which considerable variation had been observed: carriage of LESGI-5 and the prevalence of auxotrophic mutants. Nine further patients were selected and equivalent samples at the beginning and end of an exacerbation were analysed (equivalent to samples A and C) to determine if any changes had occurred over this period (Table 5). In five out of nine patients, the prevalence of LESGI-5 was $100 \%$ in both samples. In patients IV, V and $\mathrm{X}$ there was a small increase in prevalence between samples A and C (range 2.5-12.5\%). In one patient a small decrease in prevalence was observed (2.5\%; equivalent to one isolate from 40).

The prevalence of LESGI-5 carriage amongst isolates changed dramatically over the three samples for patient I, with an increase from 10 to $97.5 \%$. This striking shift could suggest positive selection for this genomic island during the period of the study. We sought evidence that this selection may occur in other patients. However, our efforts were generally confounded by the high levels of carriage occurring prior to antimicrobial therapy in many of the other patients tested. It is interesting to note that a mutation in this genomic island leads to an impaired ability to establish chronic infection in the rat chronic lung infection model (Winstanley et al., 2009), suggesting a possible role for this island in virulence. However, further investigation is needed in order to elucidate the precise role of this island, and we cannot rule out the possibility that the carriage of LESGI-5 by a subpopulation in patient I was not a significant factor in the selection of this subpopulation.

The prevalence of auxotrophic mutants in samples from each of the additional patients was highly variable $(0-$ $90 \%$ ). In samples from two patients (IX and XII) no change was observed. In samples from four patients the prevalence decreased, whereas in samples from three patients the prevalence increased (Table 5). However, percentage changes greater than $30 \%$ were only observed for three of the additional patients tested (VI, VII and XI).

Overall, in 5 out of the 12 patients studied there was a notable increase in the prevalence of auxotrophic mutants during the sampling period. The amino acid concentration

Table 5. Prevalence of LESGI-5 and auxotrophic mutants amongst LES isolates

The percentage of PCR isolates positive for the presence of auxotrophic mutants and LESGI-5 amongst sets of 40 LES isolates from 9 CF patients are shown for sputum samples taken at the beginning of an exacerbation (sample A) and taken at end of the exacerbation (sample C).

\begin{tabular}{|lccccc|}
\hline \multirow{2}{*}{ Patient } & \multicolumn{2}{c}{ LESGI-5 } & & \multicolumn{2}{c|}{ Auxotrophy } \\
\cline { 2 - 3 } \cline { 5 - 6 } & $\begin{array}{c}\text { Sample A } \\
(\%)\end{array}$ & $\begin{array}{c}\text { Sample C } \\
(\%)\end{array}$ & & $\begin{array}{c}\text { Sample A } \\
(\%)\end{array}$ & $\begin{array}{c}\text { Sample C } \\
(\%)\end{array}$ \\
\hline IV & 87.5 & 92.5 & & 32.5 & 30 \\
V & 0 & 12.5 & & 60 & 52.5 \\
VI & 100 & 100 & & 10 & 47.5 \\
VII & 100 & 100 & & 0 & 32.5 \\
VIII & 100 & 97.5 & & 0 & 7.5 \\
IX & 100 & 100 & & 0 & 0 \\
X & 0 & 2.5 & & 12.5 & 0 \\
XI & 100 & 100 & & 90 & 52.5 \\
XII & 100 & 100 & & 72.5 & 72.5 \\
& & & & & \\
\hline
\end{tabular}


of sputum is high, especially during infective exacerbations, and the concentration correlates with pulmonary disease severity (Thomas et al., 2000). It has been proposed that auxotrophy may be an advantage due to the downregulation of some metabolic pathways as the production of amino acids, in terms of energy, is expensive. This may explain the increases in the proportion of auxotrophic mutants observed in some patients.

\section{Detection of LES phages directly from the sputum of CF patients}

PCR assays were performed directly on Sputasol-treated, diluted, filter-sterilized and DNase I-treated sputum samples from patients I-III. Original testing using oligonucleotide primers specific for the LES phages produced only very weak amplicons and therefore nested primers were used to increase sensitivity. For the novel LES phages 2 and 3, primers for detection of the circular form of the genome were used. PCR assays for the oprL and 16S rRNA genes were used on all samples to ensure that there was no contamination with genomic DNA. LES phage 2 was detected in all of the sputum samples except one (IIIA), and LES phage 3 was detected in all samples except two (IB and IIIA). LES phage 4 was detected in every sample, whereas LES phage 5 was absent in all the samples except one (IIA) (Table 6).

Although LES prophage 5 was not detected in any of the isolates, this phage was detected in one of the sputum samples. We have reported previously that LES prophage 5 is carried by a minority of isolates of the LES and have noted that other LES prophages can also be unstable (Winstanley et al., 2009). However, our observations here suggest that even when LES prophage 5 cannot be detected in a screen of isolates, the phage may nevertheless be present within the LES population in sputum, albeit at a very low level. Our inability to detect greater variation in the carriage of prophages may be due to the fact that only 40 isolates were analysed per sample.

Our observations with respect to free phages in CF patient sputum samples are consistent with the possibility that antimicrobial therapy contributes to phage induction. A previous study has suggested that antibiotics commonly used in the treatment of CF patients can cause phage induction in bacteria (Rolain et al., 2009). Furthermore, hydrogen peroxide has also been shown to induce lysogenic prophages (Selva et al., 2009). The mobility of these bacteriophages could allow genetic information to be shared amongst isolates in the population. Natural phages from $P$. aeruginosa have been found to transduce antibiotic resistance (Blahova et al., 2000). In addition, the phage F116 (sharing some similarity with LES prophage 2 and 3) has been found to transduce resistance to imipenem, cefotaxime, kanamycin and carbenicillin (Blahova et al., 1994). Our observations, coupled with those reported in a previous study implicating LES phages in the competitiveness of the strain in a rat chronic respiratory infection
Table 6. Presence of free LES phages in CF patient sputum samples

The presence of four $P$. aeruginosa LES phages was determined using PCR assays performed directly on Sputasol-treated, diluted, filtersterilized and DNase I-treated sputum samples representing samples taken at the beginning of the exacerbation (sample A), samples taken during the exacerbation and antibiotic treatment (sample B), samples taken at the end of the exacerbation (sample C). Nested primers were used. +, PCR-positive; -, PCR-negative.

\begin{tabular}{|lccccc|}
\hline Patient & Sample & Phage 2 & Phage 3 & Phage 4 & Phage 5 \\
\hline I & A & + & + & + & - \\
& B & + & - & + & - \\
& C & + & + & + & - \\
II & A & + & + & + & + \\
& B & + & + & + & - \\
& C & + & + & + & - \\
III & A & - & - & + & - \\
& B & + & + & + & - \\
& C & + & + & + & - \\
& & & & & \\
\hline
\end{tabular}

model (Winstanley et al., 2009), suggest that the general role of bacteriophages in infections in the CF lung merits further investigation.

\section{Consequences of population diversity for routine diagnostic practice}

This study questions routine diagnostic laboratory practice whereby one or two isolates are chosen according to their colony morphology and antimicrobial susceptibility testing is carried out on these isolates. Our study shows that each population carries a large amount of variation in antimicrobial susceptibility and other phenotypes, and that this includes differences between isolates sharing the same colony morphology. Variations in antimicrobial susceptibility results for sputum samples both within and between laboratories have been observed by Foweraker et al. (2005). Furthermore, it has been widely reported that antibiotic susceptibility tests are poorly predictive of clinical outcomes in chronic $P$. aeruginosa infection in $\mathrm{CF}$, using either aerosolized or intravenous therapy (LiPuma, 2001; Smith et al., 2003). It has also been shown that reducing the number of routine tests has no impact on short-term clinical outcomes (Etherington et al., 2008). Therefore, in the context of CF sputum samples, it is difficult to defend the practise of choosing one or two isolates to represent a whole population of $P$. aeruginosa, and basing therapeutic decisions on the antibiotic susceptibilities derived from this.

In addition, we cannot claim that a sputum sample is entirely representative of the lung population. However, sputum analysis is widely used in medical microbiology for respiratory pathogens, and especially in the context of CF. Hence, we contend that the approach we have taken is 
enough to give us a snapshot of the LES populations in the CF lung environment.

Overall, our observations indicate that changes occur in the make-up of the $P$. aeruginosa LES populations during short periods of intense selective pressure, demonstrating the versatility and adaptability of this organism. Each set of 40 isolates was a mixture of LES subtypes; phenotypic variability was found within each sputum sample for all three patients. Every sample contained variation with respect to colony morphology, virulence factor production and antimicrobial susceptibility profiles. Even though our study tested only three patients, it seems likely that similar variations within $P$. aeruginosa are a general feature of chronically infected CF patients. A larger study, following sequential samples from multiple patients, should enable an improved understanding of the dynamics of evolving $P$. aeruginosa populations. We conclude that the 'metacommunity' needs to be considered when dealing with both the study and the treatment of chronic $P$. aeruginosa infection in CF patients.

\section{ACKNOWLEDGEMENTS}

Some of this work was undertaken under the framework of the UK Cystic Fibrosis Microbiology Consortium, an initiative funded by the Big Lottery Fund in association with the Cystic Fibrosis Trust. The authors thank the National Institute of Health Research and the North-West Development Agency for infrastructural and project support. We also acknowledge funding from the Dr Hadwen Trust for Humane Research.

\section{REFERENCES}

Al-Aloul, M., Crawley, J., Winstanley, C., Hart, C. A., Ledson, M. J. \& Walshaw, M. J. (2004). Increased morbidity associated with chronic infection by an epidemic Pseudomonas aeruginosa strain in CF patients. Thorax 59, 334-336.

Andrews, J. M. for the BSAC Working Party on Susceptibility Testing (2001). BSAC standardized disc susceptibility testing method. J Antimicrob Chemother 48 (Suppl. S1), 43-57.

Blahova, J., Hupkova, M. \& Krcmery, V., Sr (1994). Phage F-116 transduction of antibiotic resistance from a clinical isolate of Pseudomonas aeruginosa. J Chemother 6, 184-188.

Blahova, J., Kralikova, K., Krcmery, V. \& Jezek, P. (2000). Lowfrequency transduction of imipenem resistance and high-frequency transduction of ceftazidime and aztreonam resistance by the bacteriophage AP-151 isolated from a Pseudomonas aeruginosa strain. J Chemother 12, 482-486.

Boles, B. R. \& Singh, P. K. (2008). Endogenous oxidative stress produces diversity and adaptability in biofilm communities. Proc Natl Acad Sci U S A 105, 12503-12508.

Cabral, D. A., Loh, B. A. \& Speert, D. P. (1987). Mucoid Pseudomonas aeruginosa resists nonopsonic phagocytosis by human neutrophils and macrophages. Pediatr Res 22, 429-431.

Cheng, K., Smyth, R. L., Govan, J. R., Doherty, C., Winstanley, C., Denning, N., Heaf, D. P., van Saene, H. \& Hart, C. A. (1996). Spread of $\beta$-lactam-resistant Pseudomonas aeruginosa in a cystic fibrosis clinic. Lancet 348, 639-642.

Ciofu, O., Riis, B., Pressler, T., Poulsen, H. E. \& Høiby, N. (2005). Occurrence of hypermutable Pseudomonas aeruginosa in cystic fibrosis patients is associated with the oxidative stress caused by chronic lung inflammation. Antimicrob Agents Chemother 49, 22762282.

De Vos, D., Lim, A., Jr, Pirnay, J. P., Duinslaeger, L., Revets, H., Vanderkelen, A., Hamers, R. \& Cornelis, P. (1997). Analysis of epidemic Pseudomonas aeruginosa isolates by isoelectric focusing of pyoverdine and RAPD-PCR: modern tools for an integrated antinosocomial infection strategy in burn wound centres. Burns 23, 379386.

Etherington, C., Hall, M., Conway, S., Peckham, D. \& Denton, M. (2008). Clinical impact of reducing routine susceptibility testing in chronic Pseudomonas aeruginosa infections in cystic fibrosis. J Antimicrob Chemother 61, 425-427.

Fothergill, J. L., Panagea, S., Hart, C. A., Walshaw, M. J., Pitt, T. L. \& Winstanley, C. (2007). Widespread pyocyanin over-production among isolates of a cystic fibrosis epidemic strain. BMC Microbiol 7, 45 .

Foweraker, J. E., Laughton, C. R., Brown, D. F. \& Bilton, D. (2005). Phenotypic variability of Pseudomonas aeruginosa in sputa from patients with acute infective exacerbation of cystic fibrosis and its impact on the validity of antimicrobial susceptibility testing. J Antimicrob Chemother 55, 921-927.

Goss, C. H. \& Burns, J. L. (2007). Exacerbations in cystic fibrosis 1: epidemiology and pathogenesis. Thorax 62, 360-367.

Govan, J. R. \& Fyfe, J. A. (1978). Mucoid Pseudomonas aeruginosa and cystic fibrosis: resistance of the mucoid from to carbenicillin, flucloxacillin and tobramycin and the isolation of mucoid variants in vitro. J Antimicrob Chemother 4, 233-240.

Guzel, C. B. \& Gerceker, A. A. (2008). In vitro activities of various antibiotics, alone and in combination with colistin methanesulfonate, against Pseudomonas aeruginosa strains isolated from cystic fibrosis patients. Chemotherapy 54, 147-151.

Hart, C. A. \& Winstanley, C. (2002). Persistent and aggressive bacteria in the lungs of cystic fibrosis children. Br Med Bull 61, 81-96.

Hyatt, J. M. \& Schentag, J. J. (2000). Pharmacodynamic modeling of risk factors for ciprofloxacin resistance in Pseudomonas aeruginosa. Infect Control Hosp Epidemiol 21, S9-S11.

Kessler, E., Safrin, M., Blumberg, S. \& Ohman, D. E. (2004). A continuous spectrophotometric assay for Pseudomonas aeruginosa LasA protease (staphylolysin) using a two-stage enzymatic reaction. Anal Biochem 328, 225-232.

LiPuma, J. J. (2001). Microbiological and immunologic considerations with aerosolized drug delivery. Chest 120, 118S-123S.

Macia, M. D., Mena, A., Borrell, N., Perez, J. L. \& Oliver, A. (2007). Increased susceptibility to colistin in hypermutable Pseudomonas aeruginosa strains from chronic respiratory infection. Antimicrob Agents Chemother 51, 4531-4532.

Mathee, K., Ciofu, O., Sternberg, C., Lindum, P. W., Campbell, J. I., Jensen, P., Johnsen, A. H., Givskov, M., Ohman, D. E. \& other authors (1999). Mucoid conversion of Pseudomonas aeruginosa by hydrogen peroxide: a mechanism for virulence activation in the cystic fibrosis lung. Microbiology 145, 1349-1357.

McCallum, S. J., Gallagher, M. J., Corkill, J. E., Hart, C. A., Ledson, M. J. \& Walshaw, M. J. (2002). Spread of an epidemic Pseudomonas aeruginosa strain from a patient with cystic fibrosis (CF) to non-CF relatives. Thorax 57, 559-560.

McGrath, L. T., Mallon, P., Dowey, L., Silke, B., McClean, E., McDonnell, M., Devine, A., Copeland, S. \& Elborn, S. (1999). Oxidative stress during acute respiratory exacerbations in cystic fibrosis. Thorax 54, 518-523.

Mohan, K., Fothergill, J. L., Storrar, J., Ledson, M. J., Winstanley, C. \& Walshaw, M. J. (2008). Transmission of Pseudomonas aeruginosa 
epidemic strain from a patient with cystic fibrosis to a pet cat. Thorax 63, 839-840.

Montanari, S., Oliver, A., Salerno, P., Mena, A., Bertoni, G., Tümmler, B., Cariani, L., Conese, M., Döring, G. \& Bragonzi, A. (2007). Biological cost of hypermutation in Pseudomonas aeruginosa strains from patients with cystic fibrosis. Microbiology 153, 1445-1454.

Moriarty, T. F., McElnay, J. C., Elborn, J. S. \& Tunney, M. M. (2007). Sputum antibiotic concentrations: implications for treatment of cystic fibrosis lung infection. Pediatr Pulmonol 42, 1008-1017.

Nakajima, A., Sugimoto, Y., Yoneyama, H. \& Nakae, T. (2002). Highlevel fluoroquinolone resistance in Pseudomonas aeruginosa due to interplay of the MexAB-OprM efflux pump and the DNA gyrase mutation. Microbiol Immunol 46, 391-395.

Niga, T., Ito, H., Oyamada, Y., Yamagishi, J., Kadono, M., Nishino, T., Gotoh, N. \& Inoue, M. (2005). Cooperation between alteration of DNA gyrase genes and over-expression of MexB and MexX confers high-level fluoroquinolone resistance in Pseudomonas aeruginosa strains isolated from a patient who received a liver transplant followed by treatment with fluoroquinolones. Microbiol Immunol 49, 443-446.

Oliver, A. (2004). Carbapenem resistance and Acinetobacter baumannii. Enferm Infecc Microbiol Clin 22, 259-261.

Oliver, A., Canton, R., Campo, P., Baquero, F. \& Blazquez, J. (2000). High frequency of hypermutable Pseudomonas aeruginosa in cystic fibrosis lung infection. Science 288, 1251-1254.

Parsons, Y. N., Panagea, S., Smart, C. H., Walshaw, M. J., Hart, C. A. \& Winstanley, C. (2002). Use of subtractive hybridization to identify a diagnostic probe for a cystic fibrosis epidemic strain of Pseudomonas aeruginosa. J Clin Microbiol 40, 4607-4611.

Plasencia, V., Borrell, N., Macia, M. D., Moya, B., Perez, J. L. \& Oliver, A. (2007). Influence of high mutation rates on the mechanisms and dynamics of in vitro and in vivo resistance development to single or combined antipseudomonal agents. Antimicrob Agents Chemother 51, 2574-2581.

Regelmann, W. E., Elliott, G. R., Warwick, W. J. \& Clawson, C. C. (1990). Reduction of sputum Pseudomonas aeruginosa density by antibiotics improves lung function in cystic fibrosis more than do bronchodilators and chest physiotherapy alone. Am Rev Respir Dis 141, 914-921.

Rolain, J. M., Francois, P., Hernandez, D., Bittar, F., Richet, H., Fournous, G., Mattenberger, Y., Bosdure, E., Stremler, N. \& other authors (2009). Genomic analysis of an emerging multiresistant Staphylococcus aureus strain rapidly spreading in cystic fibrosis patients revealed the presence of an antibiotic inducible bacteriophage. Biol Direct 4, 1 .

Salunkhe, P., Smart, C. H., Morgan, J. A., Panagea, S., Walshaw, M. J., Hart, C. A., Geffers, R., Tummler, B. \& Winstanley, C. (2005). A cystic fibrosis epidemic strain of Pseudomonas aeruginosa displays enhanced virulence and antimicrobial resistance. J Bacteriol 187, 4908-4920.

Scott, F. W. \& Pitt, T. L. (2004). Identification and characterization of transmissible Pseudomonas aeruginosa strains in cystic fibrosis patients in England and Wales. J Med Microbiol 53, 609-615.

Selva, L., Viana, D., Regev-Yochay, G., Trzcinski, K., Corpa, J. M., Lasa, I., Novick, R. P. \& Penades, J. R. (2009). Killing niche competitors by remote-control bacteriophage induction. Proc Natl Acad Sci U S A 106, 1234-1238.

Skindersoe, M. E., Alhede, M., Phipps, R., Yang, L., Jensen, P. O., Rasmussen, T. B., Bjarnsholt, T., Tolker-Nielsen, T., Høiby, N. \& Givskov, M. (2008). Effects of antibiotics on quorum sensing in Pseudomonas aeruginosa. Antimicrob Agents Chemother 52, 3648-3663.

Smart, C. H., Walshaw, M. J., Hart, C. A. \& Winstanley, C. (2006). Use of suppression subtractive hybridization to examine the accessory genome of the Liverpool cystic fibrosis epidemic strain of Pseudomonas aeruginosa. J Med Microbiol 55, 677-688.

Smith, A. L., Fiel, S. B., Mayer-Hamblett, N., Ramsey, B. \& Burns, J. L. (2003). Susceptibility testing of Pseudomonas aeruginosa isolates and clinical response to parenteral antibiotic administration: lack of association in cystic fibrosis. Chest 123, 1495-1502.

Smith, E. E., Buckley, D. G., Wu, Z., Saenphimmachak, C., Hoffman, L. R., D'Argenio, D. A., Miller, S. I., Ramsey, B. W., Speert, D. P. \& other authors (2006). Genetic adaptation by Pseudomonas aeruginosa to the airways of cystic fibrosis patients. Proc Natl Acad Sci U S A 103, 8487-8492.

Thomas, S. R., Ray, A., Hodson, M. E. \& Pitt, T. L. (2000). Increased sputum amino acid concentrations and auxotrophy of Pseudomonas aeruginosa in severe cystic fibrosis lung disease. Thorax 55, 795-797.

Winstanley, C. \& Fothergill, J. L. (2009). The role of quorum sensing in chronic cystic fibrosis Pseudomonas aeruginosa infections. FEMS Microbiol Lett 290, 1-9.

Winstanley, C., Langille, M. G. I., Fothergill, J. L., Kukavica-Ibrulj, I., Paradis-Bleau, C., Sanschagrin, F., Thomson, N. R., Winsor, G. L. \& Quail, M. A. \& other authors (2009). Newly introduced genomic prophage islands are critical determinants of in vivo competitiveness in the Liverpool epidemic strain of Pseudomonas aeruginosa. Genome Res 19, 12-23. 